\title{
A Control Approach to Explore the Dynamics of Capacity Scalability in Reconfigurable Manufacturing Systems
}

\author{
Ahmed M. Deif and Waguih H. EIMaraghy, Intelligent Manufacturing Systems (IMS) Centre, Dept. of Industrial \& \\ Manufacturing Systems Engineering, University of Windsor, Windsor, Ontario, Canada
}

\begin{abstract}
This paper presents a dynamic model and analysis for one of the major characteristics of reconfigurable manufacturing systems (RMSs)-capacity scalability. The dynamic model is analyzed using its transfer function. Dynamic characteristics associated with the delay in capacity scalability and how to minimize this delay are discussed using control approaches. The problem of how to supply exact capacity in response to market changes is also examined by solving the dynamic problem of the production offset phenomenon in RMSs. The effect of work in process as a damping factor for production disturbances during capacity scalability is addressed. Finally, a general capacity scalability controller design is proposed to improve the dynamic performance of RMSs in response to sudden demand changes. The proposed controller considers the different activities associated with the capacity scalability process. A numerical example is also presented to highlight the applicability of the approach.
\end{abstract}

Keywords: Dynamics, Capacity Scalability, Control, Reconfigurable Systems

\section{Introduction}

Unpredictable and dynamic market changes cause changing requirements concerning the output capacity and variety of processing functions of manufacturing systems. Reconfigurable manufacturing systems (RMSs) can help to meet these challenges by combining the advantages of highly productive dedicated manufacturing lines (DMLs) and highly flexible manufacturing systems (FMSs). RMSs are designed to have the ability to adapt to expected or unexpected demand changes through consecutive systems' and/or system components' structure modifications, which also guarantee efficient functionality use (Urbani et al. 2001). The key characteristics of RMSs that enable these systems to achieve their goals are modularity, integrabilty, convertibility, customization, and diagnosability (Mehrabi, Ulsoy, and Koren 2000).

Ideally in RMSs, exact capacity is supplied when needed and where needed. Thus, the production capacity should be adjusted or scaled to the demand continually, so as to always be in a profitable state. However, this type of policy is undesirable or impossible due to the fact that the rate of variation in demand is usually much higher than the rate at which capacity can be changed. So the desire of following the demand has to be balanced by the risk of losses due to too-frequent changes in capacity (Asl and Ulsoy 2002a). Figure 1 shows a typical capacity scalability policy in RMSs where capacity is scaled to meet demand changes.

The capacity scalability process is determined by hard and soft activities. By hard activities is meant all the addition or reduction of the physical components of that process, such as machines, spindles, and so on. The soft activities include the managerial decisions associated with the capacity scalability process and the time required for these decision processes. In other words, these soft activities reflect the inertia of the company to react to changes. It is important to note that modeling the soft activities is very difficult due to the relationships among them, which are usually nonlinear relations. An example 


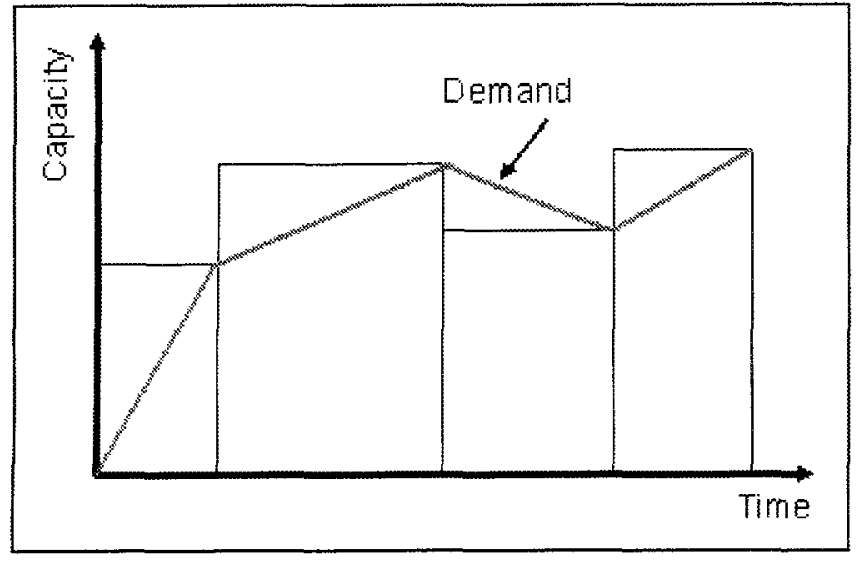

Figure I

Capacity Scalability in Reconfigurable Manufacturing Systems (RMSs)

of the hard and soft components of capacity scalability in an automatic PCB assembly line was illustrated by Deif and ElMaraghy (2004).

Manufacturing systems researchers are more and more driven to a dynamic rather than a static view of these systems (Ratering and Duffie 2003). Such a view is well justified within today's dynamic market and continuously evolving systems like RMSs. Within this scope, this paper explores the dynamics of capacity scalability in RMSs. A dynamic model is developed and a capacity scalability controller is proposed to scale the capacity in response to demand changes. Control theoretic approaches are used to analyze how exact capacity can be supplied when needed and the role of work in process (WIP) during this capacity scalability process.

\section{Literature Review}

The capacity scalability problem addresses when, where, and by how much the capacity of the manufacturing system should be optimally scaled. The problem was classically addressed from a static view as the problem of capacity expansion. The first study of capacity expansion problem is the one conducted by Manne (1967). He assumed the demand forecast is known for the next known duration of time. Also, in order to satisfy the growing demand, production capacity should be expanded over the years, and there is a cost involved with this expansion that shows substantial economies of scale. Assuming the existence of such substantial economies of scale implies the desirability of building new capacity considerably in advance of demand. But how much in advance considering the present value of future costs is an optimization problem addressed by most capacity management research afterward. Extensive review for the classical capacity expansion problem can be found in Luss (1982).

As for flexible manufacturing systems (FMSs), Leachman and Carmon (1992) proposed a procedure to generate the capacity set of alternative machine types assuming that processing time among alternative machine types is identical or proportional across the operations that they can perform. Roundy, Zhang, and Cakanyildirum (2000) considered a discrete-time capacity expansion problem in flexible manufacturing environments that can deal with multiple product families with multiple machine types and nonstationary stochastic demand. Capacity expansion decisions are made to strike an optimal balance between investment costs and lost sales costs. Liberopoulos (2002) expressed the capacity of the flexible manufacturing system in terms of the total production rates of all part types over all machines. The capacity set is expressed as a convex hull of a set of points corresponding to all possible assignment of machines to part types, where in each assignment each machine allocates all of its capacity to only one part type. The capacity scalability in flexible manufacturing systems is viewed as how can the system satisfy the demand within the existing capacities alternatives in an optimal way due to the existence of fixed but programmable machines.

In RMSs, the capacity scalability problem was viewed from a different perspective because the ability to smoothly change the existing system capacity through rearranging or changing (adding or removing) the system components became possible. Also, from a time perspective, the change in these systems is supposed to be faster with shorter ramp-up time. Son, Olsen, and Yip-Hoi (2001) suggested station paralleling within a stage as a possible approach to scale the capacity of the manufacturing system. This was proposed to solve the problem of unused capacity even if the line is totally balanced. An approach for capacity scalability management in RMSs with stochastic market demand was presented by Asl and Ulsoy (2002a), where an optimal region for the capacity scalability management policy based on Markov decision theory was presented. Also, Asl and Ulsoy (2002a) considered the time delay between the time the capacity is ordered and the time it is delivered. Situations for perfect and imperfect information about demand were examined, and the value 
of market information was suggested. Deif and ElMaraghy (2005) proposed an optimal capacity scalability scheduling approach where based on the demand and the cost of capacity scalability an optimal capacity scalability schedule at minimum cost can be generated.

Dynamic attempts to study capacity scalability problem were triggered by the application of control theory to manufacturing systems analysis by Simon (1952) and more recently Towill (1982). Asl and Ulsoy (2002b) presented a dynamic approach to capacity scalability modeling in RMSs based on the use of feedback control. The approach presented suboptimal solutions that are robust against demand variations.

The funnel model of manufacturing systems developed by Wiendahl (1995) is a dynamic model that manipulated feedback control approach with the help of logistic operating curves developed by Nyhuis (1994) to control WIP and capacity of manufacturing systems as in Wiendahl and Breithaupt $(1999,2000)$. In their approach, the capacity scalability required was found using flexibility curves, which indicate the time delay of each capacity scaling step. The capacity scalability controller is to choose the best capacity scaling decision based on the backlog value and delay acceptable.

A dynamic model developed by Duffie and Falu (2002) for closed-loop production planning and control (PPC) was proposed to control WIP and capacity. They investigated the effect of choosing different capacity scalability controller gains as well as the WIP controller gains on system performance and how this can be used to achieve required system responses. The previous work was extended by Kim and Duffie (2004) to study the effect of capacity disturbances and capacity delays on system performance. Their results highlighted the fact that if capacity can be adjusted more often with less delay, the system's performance will be improved in a changing-demand environment.

In the previous research works, the capacity scalability problem was modeled using control theoretic approaches; however, much of the dynamic analysis is still required. In particular, there is a need to model capacity scalability dynamics and explore how to solve the problems of reducing the scalability delay, supplying the required capacity, and finally damping production oscillation in response to demand changes. This paper, in addition to presenting a new dynamic model for capacity scalability, ad- dresses these problems from a dynamic perspective utilizing control design approaches.

\section{Dynamic Model for Capacity Scalability in RMS}

A dynamic model of a reconfigurable manufacturing system is constructed in which different system configurations (due to capacity scalability) can be realized in response to continuously changing demand. The modeling approach and its analysis are based on the application of control theory and feedback analysis where a continuous time domain is implemented to model the system states. Although a discrete time domain gives a better image of the manufacturing systems, the continuous Laplace models are favored in this paper because the interesting parameters (production rate, WIP level, lead time, etc.) show a more continuous character (Wiendahl and Breithaupt 1999). In other words, continuous time modeling is justified due to the level of abstraction of the model that deals with the tactical rather than the operational level. Furthermore, various manufacturing systems, like those with continuous flow, are better modeled with the continuous time approach. Finally, similar dynamic characteristics can be obtained using discrete models (John, Towill, and Naim 1994). The proposed approach has a continuous time model, while its control is a discrete-action one.

\section{Model Parameters}

The system shown in Figure 2 is composed of three main components, the production process, WIP controller, and capacity scalability controller. The production process is modeled as a pipeline where the outflow is simply lagged by the production lead time, $T_{L T}$ (Sterman 2000). Thus, the lead time is found by analogy with a pipeline of a known length into which material is fed and from which it flows once the material has passed through the pipe. This is a simplification of a complex group of interacting entities such as a manufacturing system to a single, simple function, although it results in errors when determining an optimal solution. Good designs in terms of model parameter settings (the values of time parameters and controller gains) and architecture (the placement and component makeup of the feedback paths) will reduce these errors and at the same time enable the designer to have a better dynamic analysis of the system. 


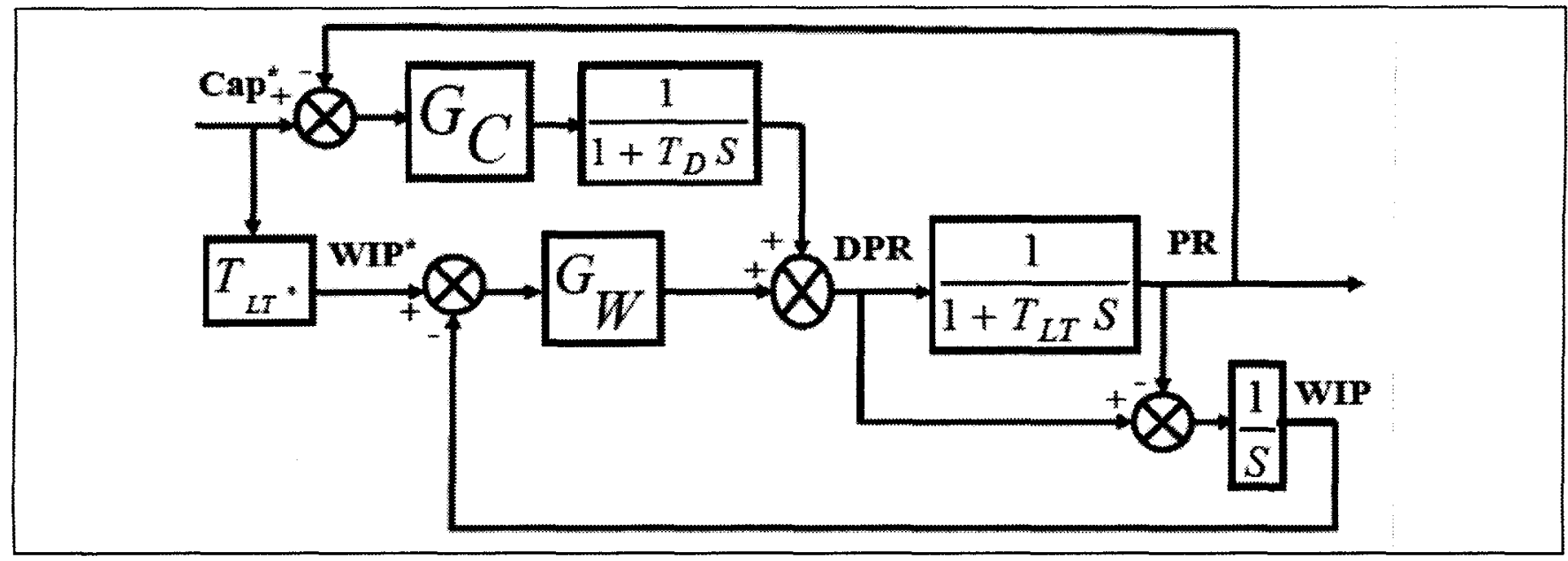

Figure 2

Block Diagram for Dynamic RMS Model

Determining the exact value of pipeline lead time is a complex task (Hoyt 1980) and is beyond the scope of this paper. However, an exponential lag model is used in the developed model that can be considered representative of batch manufacture (Towill 1982). Simulation results of such assumptions showed exponential pipeline lag to be an appropriate compromise between complexity and accuracy (Wikner 1994). More details about modeling production lead time can be found in (Wikner 2003).

Accounting for WIP in dynamic modeling of RMSs is very important as it decreases the oscillation of the system and affects the damping ratio of the system especially in the case of unanticipated shocks (e.g., rush orders). WIP is also an important control parameter as it ties up capital and costs interest and has direct relation with the production rate and production lead time. As mentioned earlier, production lead time is difficult to measure, while WIP is easy to measure, and therefore WIP can be an indicative and easy parameter to use for normal control of manufacturing systems. In reality, any manufacturing system configuration has a maximum WIP point (disregarding any space limitations) based on the requirement of a fixed lead time. The amount that WIP can be increased to impact production is thus limited. To overcome this problem and maintain the advantage of a WIP-based production control system, the system's capacity should be reconfigured (scaled). This is achieved through the new enabling technologies of RMSs. The desired WIP level is calculated as a product of multiplying the order rate, OR (which is equal to the desired capacity rate in the case of a RMS), by the estimated (ideal) lead time of the production system, $T_{L T}^{*}$, as indicated by Little's law (Sterman 1989).

In this paper, the ideal lead time is assumed to be equal to the actual lead time. Numerous researches have been conducted to relax this assumption (Towill, Evans, and Cheema 1997); however, the relaxation of this assumption is beyond the scope of this paper. The WIP control gain $\left(G_{W}\right)$ can be physically described as increasing or decreasing the input rate of work to the production system (Duffie and Falu 2002) because stocks (as in the case of WIP here) are altered only by changes in their inflow and outflow rates (Sterman 2000). The reference capacity rate is set to be equal to the order rate, OR. A suggested formula that can be used to calculate the reference capacity is shown in Eq. (1):

$$
\begin{aligned}
& \text { Cap }^{*}= \\
& \frac{\text { Demand over planning horizon (\# of goods) }}{\text { Planning horizon (days) }}
\end{aligned}
$$

Any demand disturbance will immediately be reflected on the value of Cap", and thus it is a dynamic parameter. Examples of the capacity scalability control gain $\left(G_{C}\right)$, within the RMS paradigm, are adding or removing machines, adding or removing machine tools or components, and increasing or decreasing work shifts.

The capacity scalability delay time, $T_{D}$, is important to consider when capacity scalability controllers are involved. By RMS definition, this delay tends to be zero; however, in reality it is impossible to adjust the capacity immediately (Petermann 1996). Therefore, a reaction time between the request for 
capacity and the following allocation was introduced in the model. The capacity installation/uninstallation time delay is usually a function in the hard part of the process (capacity size and type) and the associated soft part, as explained earlier. Thus, it varies based on the required capacity correction action. This delay can be used to measure the degree of reconfigurability of the manufacturing system.

In this model, the time parameters $T_{L T}$ and $T_{D}$ are set by the user; as the model operates, WIP and capacity levels are then adjusted (using the controllers gains $G_{W}$ and $G_{C}$, respectively), and system performance is measured in terms of both the production rate over time and the WIP target level.

\section{Model Operation}

The operation of the dynamic RMS model can be stated as follows. The WIP level is observed and compared to a reference WIP level. Based on the error between the two levels, the WIP controller adjusts the WIP level through a gain $\left(G_{W}\right)$ and adds this amount to the desired production rate, DPR, level. Once the system reaches the maximum WIP point, no more WIP gain can be added by the WIP controller, and thus the capacity controller is activated to eliminate the backlog by reconfiguring the system to scale up the capacity. The new system configuration will introduce a new WIP maximum point, and the system will be automatically set back to the WIPbased control mode. When the capacity scalability controller is operated, the production rate, PR, is observed and compared to a reference capacity rate. Based on the error between the two rates, the capacity scalability controller adjusts the capacity rate through a gain $\left(G_{C}\right)$ and adds this amount to the desired production rate, DPR, level.

WIP level is calculated as the difference between the desired production rate, DPR, and the actual production rate, $\mathrm{PR}$. The $\mathrm{PR}$ is due to an exponential time delay of the DPR based on the system's production lead time, $T_{L T}$ (John, Towill, and Naim 1994). The WIP controller is appropriate for normal production control below the max WIP point. If the lead time keeps growing due to any internal disturbances or if there is a rush order, the queue of waiting orders in front of the system (WIP level) can be diminished by decreasing the system's input rate through the WIP controller. However, if there is a due date limit (which is a typical case in the reconfigurable manufacturing environment) then the input rate cannot be reduced and thus capacity should be scaled up.

The capacity scalability controller functions when the maximum WIP level is reached and the input rate cannot be decreased, as otherwise a backlog does not arise. However, if the capacity is increased by the capacity controller to compensate for the undesirable WIP increase and then the system is back into the stable state, the system can be in a state of un-utilized capacity. The WIP controller will not detect this problem. Thus the capacity scalability controller will also be used to resolve this undesired situation by observing the production rate, $\mathrm{PR}$, and comparing it to a capacity reference point and then scaling down the capacity with the difference between the two levels. It is important to note that because the WIP can be adjusted much more easily than the capacity (because capacity cannot be changed in a continuous manner), the response of the WIP loop is faster than the response of the capacity loop.

The characteristic equation of the developed dynamic RMS model is derived and expressed in Eq. (2). The model has a second-degree characteristic equation, which means that it can be easily analyzed and controlled. Detailed parametric analysis and examining the effect of the different parameters on the model's undamped natural frequency and damping ratio can be found in Deif and ElMaraghy (2006). The model notations are listed in the Appendix.

$$
\begin{aligned}
& \frac{\mathrm{PR}}{\mathrm{Cap}^{*}}= \\
& \frac{G_{W}\left(T_{D}^{-1}+S\right)+G_{C} T_{L T}^{-1} T_{D}^{-1}}{S^{2}+S\left(T_{D}^{-1}+T_{L T}^{-1}+G_{W}\right)+\left(G_{W} T_{L T}+G_{C}+1\right) T_{L T}^{-1} T_{D}^{-1}}
\end{aligned}
$$

To summarize, the proposed model contains two feedback loops. The first loop is for the WIP error and the second loop is for the production rate error. These loops are dependent on each other because the controller of the second loop (capacity scalability controller) is engaged only when the controller of the first loop (WIP controller) cannot work beyond the maximum WIP limit. More analysis of this dynamic behavior of the model is discussed in the following section.

\section{Capacity Scalability Model Analysis}

This section explores different capacity scalability dynamic characteristics of the developed RMS model. 
The response of the developed characteristic equation of the model is tested against demand disturbances to reflect the real reconfigurable operation's environment. The analysis will include the effect of the capacity scalability delay on the system dynamics, the production offset problem (deviation from the target production level), the effect of the WIP controller on damping the production oscillation, and finally a comparison between the responses of different capacity scalability controller alternatives against sudden demand change. It is important to state here that the aim of this section is to get an insight about these different dynamic characteristics of RMSs, while the optimal parameters settings of the model and the ideal controllers design calculations will be explored in other research work.

\section{Examining the Effect of Capacity Scalability Delay}

Ideally, reconfigurable manufacturing systems aim to scale the capacity exactly when needed and thus theoretically there is no delay incurred in this scalability process. However, practically speaking, this is very difficult to achieve due to the different hard and soft activities associated with the scalability process. The effect of capacity scalability delay on RMS dynamics can be illustrated by comparing the response of two reconfigurable systems - one with no capacity scalability delay (limit case) and the other that incurs some delay while scaling the capacity. To achieve this comparison, the characteristic equation of the developed model expressed in Eq. (2) will be modified to eliminate the capacity delay component after the capacity scalability controller. The new characteristic equation of the no-delay RMS is shown in Eq. (3):

$$
\frac{\mathrm{PR}}{\mathrm{Cap}^{*}}=\frac{G_{W}+G_{C} T_{L T}^{-1}}{S+\left(G_{W}+G_{C} T_{L T}^{-1}+T_{L T}^{-1}\right)}
$$

The responses of both systems to a sudden change in the demand are shown in Figure 3 (represented by a step change). The system parameters were assumed arbitrarily to be as follows: lead time $=5$ days, capacity scalability delay time $=3$ days, WIP control gain $=1$, and capacity scalability control gain = 7. The results shown in Figure 3 show that the manufacturing system with no capacity scalability delay has a shorter rise time, indicating that it is more responsive to demand change. This can be easily un-

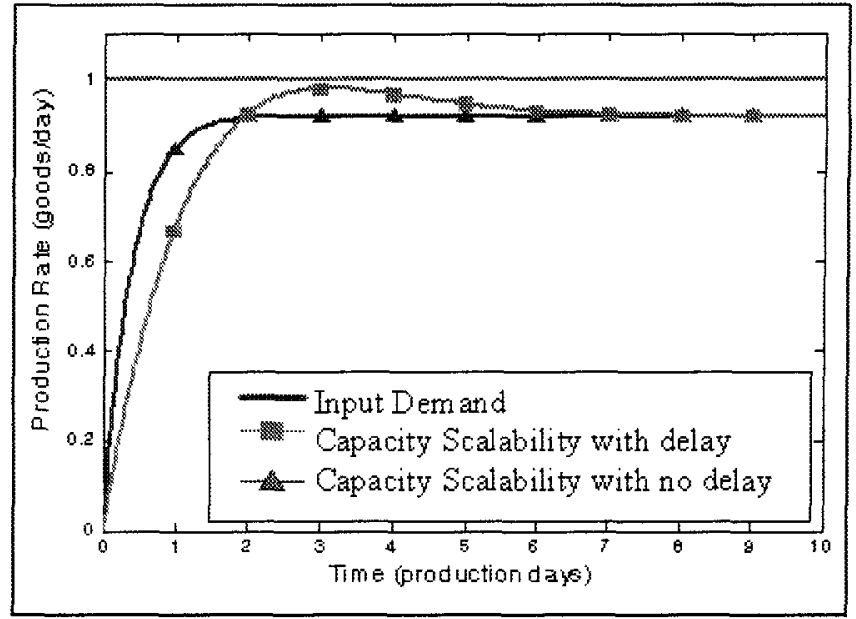

Figure 3

Dynamic RMS Model Responses With and Without Capacity Scalability Delay

derstood due to the time difference between the two systems caused by the capacity scalability delay. A second observation that can be realized is the existence of a production offset from the required demand. This problem will be discussed and solved in the next section. Another important fact is the presence of an overshoot in production only in the system with capacity scalability delay. This can be explained using control theory by realizing that the system with no capacity scalability delay is a firstorder system while the system with capacity scalability delay is a second-order system. Also, it can be related to the fact that any delay in the causal link of the negative feedback loop will lead to overshoot and oscillation. From a manufacturing standpoint, the overshoot happens due to the desire of responding quickly to the sudden demand change.

A conclusion that can be derived from this fact is that capacity scalability delay plays an important role in the reconfigurable manufacturing system dynamics by causing an overshoot in the production of these systems when they are exposed to market disturbances. To overcome the production overshooting problem in RMSs, a new capacity scalability controller design is suggested. The new design will include a derivative component to change the controller type from proportional controller, $\mathrm{P}$, to a proportional and derivative controller, PD. The new control gain law of the capacity scalability controller will be $G c=G c$ $(1+b S)$, where $b$ is the derivative controller gain. From a manufacturing point of view, the derivative part accounts for the extra time required for installing the extra capacity (which is indicated by the propor- 
tional gain) and the time for new system configuration to ramp up. The new characteristic equation for the developed manufacturing model after augmenting this ideal derivative compensator to the capacity scalability controller is shown in Eq. (4):

$$
\begin{aligned}
& \frac{\mathrm{PR}}{\mathrm{Cap}^{*}}= \\
& \frac{S\left(G_{W}+G_{C} b T_{L T}^{-1} T_{D}^{-1}\right)+\left(G_{W} T_{L T}+G_{C}\right) T_{L T}^{-1} T_{D}^{-1}}{\left[\begin{array}{c}
S^{2}+S\left(T_{D}^{-1}+T_{L T}^{-1}+G_{W}+G_{C} b T_{L T}^{-1} T_{D}^{-1}\right) \\
+\left(G_{W} T_{L T}+G_{C}+1\right) T_{L T}^{-1} T_{D}^{-1}
\end{array}\right]}
\end{aligned}
$$

To examine the effect of the new controller design on the transient response of the manufacturing system (production overshoot), the response of both systems (with P and PD capacity scalability controllers) with different scalability delay values will be plotted against a step change in market demand. The same system parameters used in the previous simulation will be used except for setting $b=1$ and varying the delay time. The results are shown in Figures $4 a$ and $4 b$. The analysis of both figures reveals that the transient responses of the manufacturing system with the PD controller are much more improved than those of the system with the $\mathrm{P}$ controller, indicating a decrease in the production overshooting problem when demand is suddenly altered. Another observation that can also be drawn from Figure $4 a$ is that as the scalability delay time increases the amount of production overshoot decreases. This highlights the trade-off decision that should be made to balance between the responsiveness of the system and the production overshooting problem. This decision will be reflected in the values of the parameters settings, especially the derivative controller gain, $b$. However, as previously indicated, the optimal parameters setting of the developed RMS model will be explored in further research.

\section{Solving the Production Offset Problem}

As seen from previous results, there was always a production offset problem with every response to any demand change. From a manufacturing point of view, the offset in production from the required target level is due to the different nonlinearities involved in the manufacturing process, such as the various soft decisions and different time delays. Mathematically, the reason for such a problem can be explained as fol-
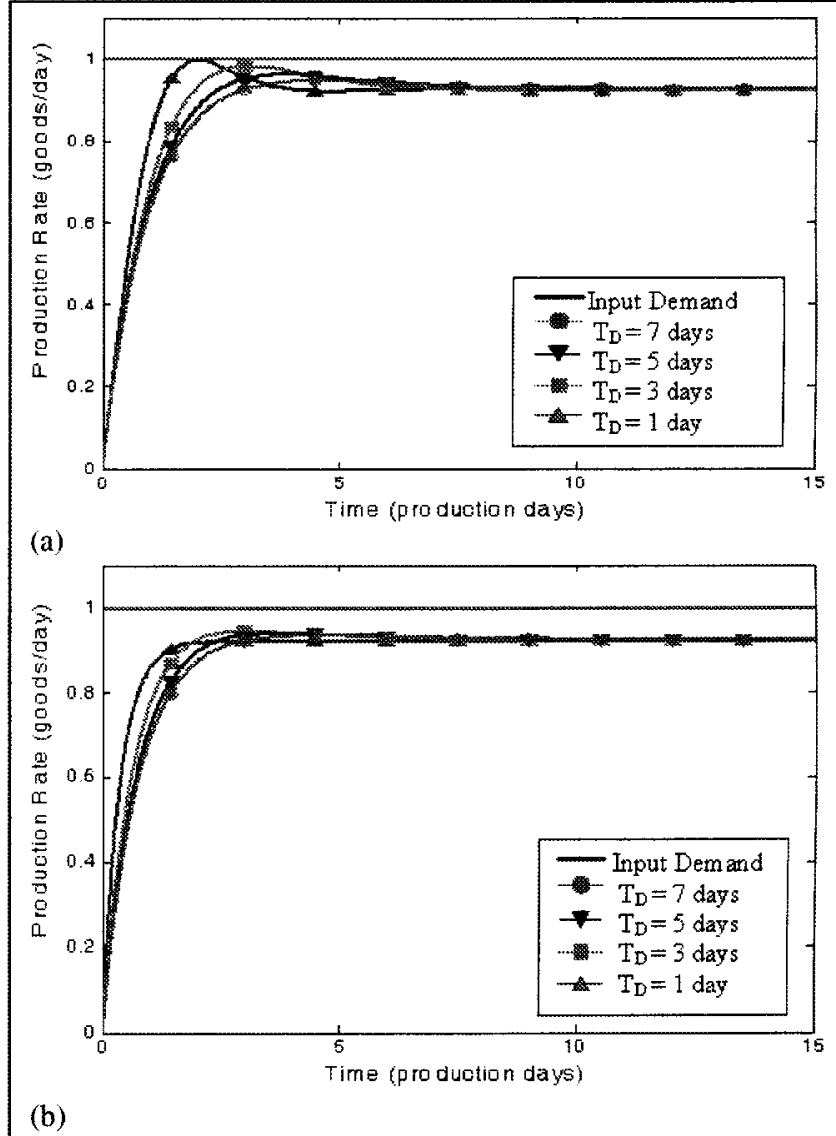

Figure 4

Dynamic RMS Model Responses with Capacity Scalability Delay (a) with a $P$ Controller and (b) with a PD Controller

lows: The objective of RMSs is to have the production exactly equal the demand in order to have the best performance in terms of responsiveness. This implies aiming to reach the state described in Eq. (5):

$$
\begin{aligned}
& \mathrm{PR}=\mathrm{Cap}^{*} \\
& \text { (since Cap }{ }^{*} \text { directly reflects } \\
& \text { the demand as stated in Eq. 1) }
\end{aligned}
$$

However, in the developed model, if the WIP controller is eliminated, then the desired production rate, DPR, will be equal to:

$$
\begin{aligned}
& \mathrm{DPR}=\left(\mathrm{Cap}^{*}-\mathrm{PR}\right) G_{C} \\
& \mathrm{PR}=\mathrm{Cap}^{*}-\mathrm{DPR} / G_{C}
\end{aligned}
$$

From Eqs. (5) and (7),

$$
\mathrm{PR}=\mathrm{PR}-\mathrm{DPR} / G_{c}
$$

It is clear from Eq. (8) that when exact chasing of demand RMS policy is targeted and if there is no WIP 
compensation involved in the system, there will be a production offset or drifts in the state of equilibrium equal to DPR $/ G_{C}$. From the developed structure, one of the solutions that can be suggested to this problem is to adjust the WIP control gain value so that it does not only compensate for the difference between the target WIP level and the actual WIP level, but also compensates for this production offset. However, this optimal solution for the design of the WIP gain, $G_{W}$, is not always feasible due to the limitation on the values of the WIP gain, as explained earlier.

In this paper, the solution proposed for this problem is to redesign the capacity scalability controller to include an integral gain to eliminate the production offset. The new control gain law of the capacity scalability controller will be $G c=G c(1+a / S)$, where $a$ is the integral controller gain. The role of this integral gain is to provide the capacity controller with a better ability to follow the target level (tracking). This happens through the "accumulating" action of such a component. In the manufacturing context, this means that the controller will increase the scaled capacity beyond the difference between the current production rate and the target capacity rate with an amount that accommodates for the nonlinearities explained earlier in the process.

The new characteristic equation of the developed manufacturing model after augmenting this ideal integral compensator to the capacity scalability controller is shown in Eq. (9). The new equation is of higher order, which indicates that a greater effort is to be made to control the new system and, in turn, to eliminate the offset.

$$
\begin{aligned}
& \frac{\mathrm{PR}}{\mathrm{Cap}^{*}}= \\
& \frac{S^{2} G_{W}+S\left(G_{W} T_{D}^{-1}+G_{C} T_{L T}^{-1} T_{D}^{-1}\right)+G_{C} a T_{L T}^{-1} T_{D}^{-1}}{S^{3}+S^{2}\left(T_{D}^{-1}+T_{L T}^{-1}+G_{W}\right)} \\
& {\left[\begin{array}{c}
+S\left(G_{W} T_{L T}+G_{C}+1\right) T_{L T}^{-1} T_{D}^{-1}+\left(G_{C} a T_{L T}^{-1} T_{D}^{-1}\right)
\end{array}\right]}
\end{aligned}
$$

To examine the effect of the new controller on eliminating the production offset problem, the responses of both systems (with P and PI capacity scalability controllers) at different capacity scalability and WIP gains values $\left(G_{C}\right.$ and $\left.G_{W}\right)$ will be plotted against a step change in the market demand. The same parameters settings will be used and the integral gain $a=2$. The results are shown in Figures $5 a$
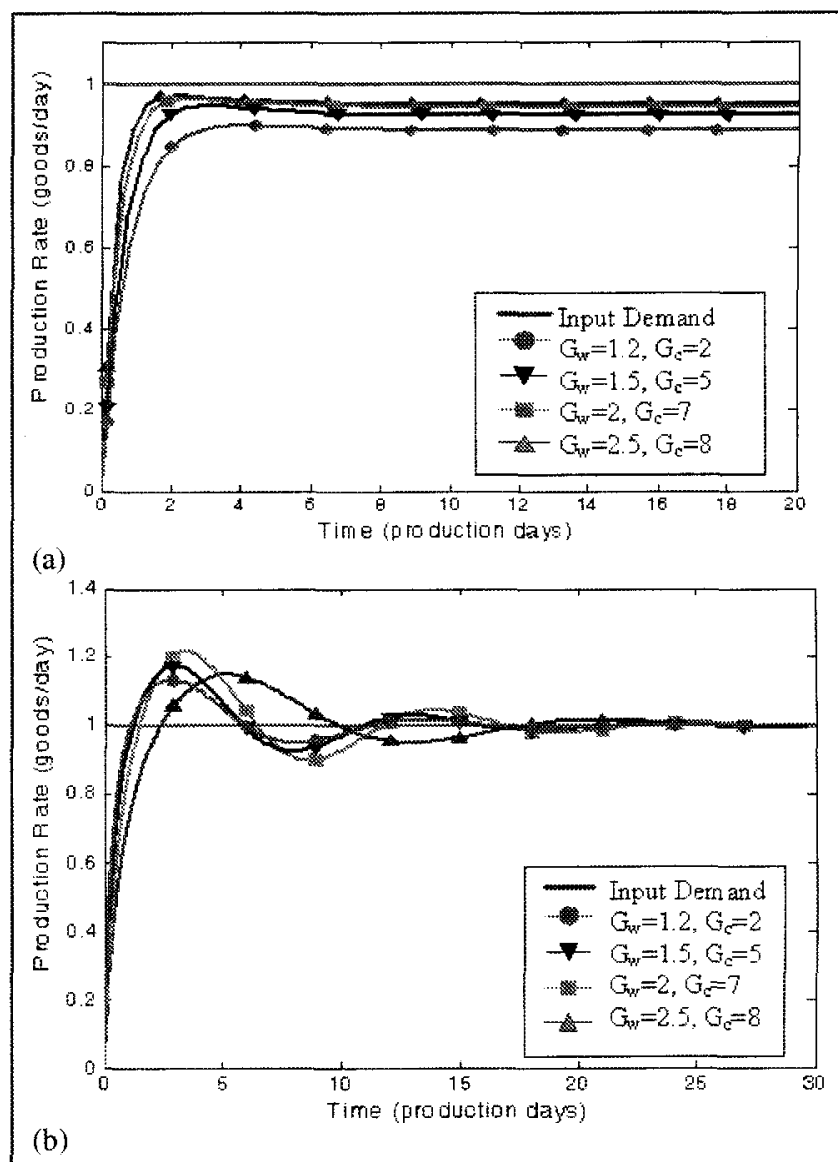

Figure 5

Production Offset in Dynamic RMS Model

(a) with a $P$ Controller and (b) with a PI Controller

and $5 b$. The analysis of Figure $5 a$ shows that even if both controllers' gains are raised, the production offset problem will still exist. This problem disappeared in Figure $5 b$ due to the existence of the new PI capacity controller design. Figure $5 b$ shows also the inherent destabilization effect of using integral control that appears in the overshooting and oscillation of the system before reaching the desired production rate. This problem is managed by the proportional controllers and it decreases as the values of both gains $G_{C}$ and $G_{W}$ increase.

It should be noted, based on control theory, that the time required to reach the target state is determined by the ratio of the proportional to the integral time, which highlights the importance of studying the optimal parameters settings for the developed model.

Another reason that can lead to a production offset in the developed model will be a difference between the expected lead time, $T_{L T}^{*}$, and the actual lead time, $T_{L T}$. This was proved by Disney and Towill (2005) through applying the final value theorem to 
their dynamic model, which also expressed the production process using pipeline delay. The reason for the offset in that case will be because the desired WIP level is based on the perception of the production lead time, and the actual WIP will be based on the actual production lead time. This case is not addressed in this paper as both lead times are assumed to be equal.

\section{Effect of WIP Controller on Damping Production Oscillation}

As shown in the previous section, solving the offset problem was at the expense of having some production oscillation. To decrease such a problem, a damping factor should be involved. In the developed RMS model, WIP is the damping parameter for the manufacturing system, and its damping effect increases as the WIP gain value, $G_{W}$, increases. From a mathematical point of view, this can be explained by examining the damping ratio in the characteristic equation of the developed model. As shown in Eq. (10), the only controllable factor that can increase the damping ratio, $\zeta$, and thus decrease the oscillation, is the WIP control gain, $G_{W}$, while both the WIP control gain, $G_{W}$, and the capacity scalability control gain, $G_{C}$, affect the natural frequency, $\omega_{n}$, of the manufacturing system.

$$
\begin{aligned}
& \zeta=\frac{1}{2 \omega_{n}}\left(\frac{1}{T_{L T}}+\frac{1}{T_{D}}+G_{W}\right) \\
& \text { where } \omega_{n}=\sqrt{\frac{G_{W} T_{L T}+G_{C}+1}{T_{L T} T_{D}}}
\end{aligned}
$$

This also can be explained because fully accounting for work in process will convert the potentially oscillatory negative production loop with lead-time delay into an effectively first-order negative feedback system. From a manufacturing standpoint, this is achieved because the WIP controller will compensate for production during the delay incurred due to capacity scalability. The effect of the WIP controller, $G_{W}$, on damping the production oscillation and how the oscillation decreases with increasing $G_{W}$ is shown in Figure 6.

\section{Comparing Different Capacity Scalability Controllers}

It is difficult to specify an optimal capacity scalability controller or a best capacity scalability policy. This is because there are different trade-off decisions that should be taken while picking a certain capacity scalability controller design. These trades-offs are usually dependent on the enterprise market strategy and other related hard and soft capacity scalability parameters. However, based on the previous analysis, one can claim that each controller type discussed solved some of the problems incurred in the process of capacity scalability in RMS.

The proportional, $\mathrm{P}$, controller is responsible mainly for scaling the capacity to meet the demand by correcting the difference between the production rate and the desired capacity rate (the demand); however, there will be an overshoot due to capacity scalability delay and also a production drift or offset. The proportional plus derivative, $\mathrm{PD}$, controller can help in decreasing the overshoot in production due to capacity scalability delay, while the offset problem will remain. The proportional plus integral, PI, controller is used to compensate for the production offset and other nonlinear parameters during the capacity scalability process without accounting for any overshoot due to capacity scalability time delay. Thus, a capacity scalability controller that can be suggested to include all previous advantages and overcome the stated shortcomings is the proportional plus derivative plus integral, PID, controller. The new characteristic equation of the developed model after augmenting the new PID controller to the system is shown in Eq. (11):

$$
\begin{aligned}
& \frac{\text { PR }}{\text { Cap }^{*}}= \\
& \frac{\left[\begin{array}{c}
S^{2}\left(G_{W}+G_{C} b T_{L T}^{-1} T_{D}^{-1}\right)+S\left(G_{W} T_{D}^{-1}+G_{C} T_{L T}^{-1} T_{D}^{-1}\right) \\
+G_{C} a T_{L T}^{-1} T_{D}^{-1}
\end{array}\right]}{\left[\begin{array}{c}
S^{3}+S^{2}\left(T_{D}^{-1}+T_{L T}^{-1}+G_{W}+G_{C} b T_{L T}^{-1} T_{D}^{-1}\right) \\
+S\left(G_{W} T_{L T}+G_{C}+1\right) T_{L T}^{-1} T_{D}^{-1}+\left(G_{C} a T_{L T}^{-1} T_{D}^{-1}\right)
\end{array}\right]}
\end{aligned}
$$

To compare between the different controllers discussed, the response of each controller to a step change in demand is plotted in Figure 7 . The production lead time, $T_{L T}$, is set to five days, the capacity scalability delay, $T_{D}$, is three days, the WIP gain, $G_{W}=2$, the capacity proportional gain, $G_{C}=7$, the integral gain, $a=2$, and the derivative gain, $b=2$.

The results illustrate the previous discussion about each controller and show the superiority of the PID capacity scalability controller over other controllers. 


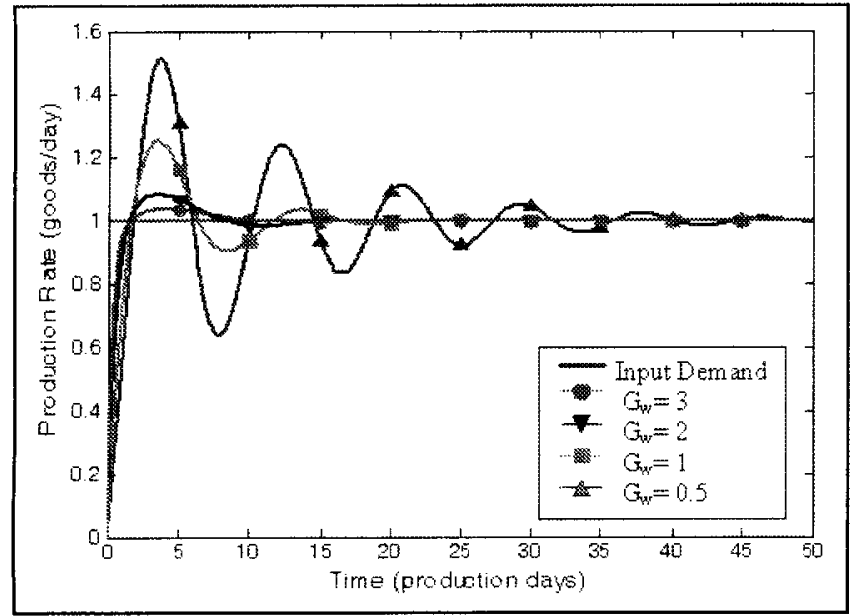

Figure 6

Effect of WIP Controller on Damping Production Oscillation During Capacity Scalability

However, the PID controller still incurs some unavoidable problems during the transient response that should be tackled by control design approaches in accordance to the specification of each enterprise.

\section{Numerical Example}

To illustrate the proposed dynamic approach, a simple numerical example of a reconfigurable machine shop that has a capacity of $40 \mathrm{~K}$ goods per day is simulated. The line has $95 \%$ utilization level and a lead time of one day $\left(T_{L T}=1\right)$. The demand is subject to various changes due to market fluctuations. Capacity can be scaled up to $10 \%$ of its current value through adding extra machines to the shop within two days $\left(T_{D}=2\right)$. The proposed capacity scalability dynamic model is compared with a classical,

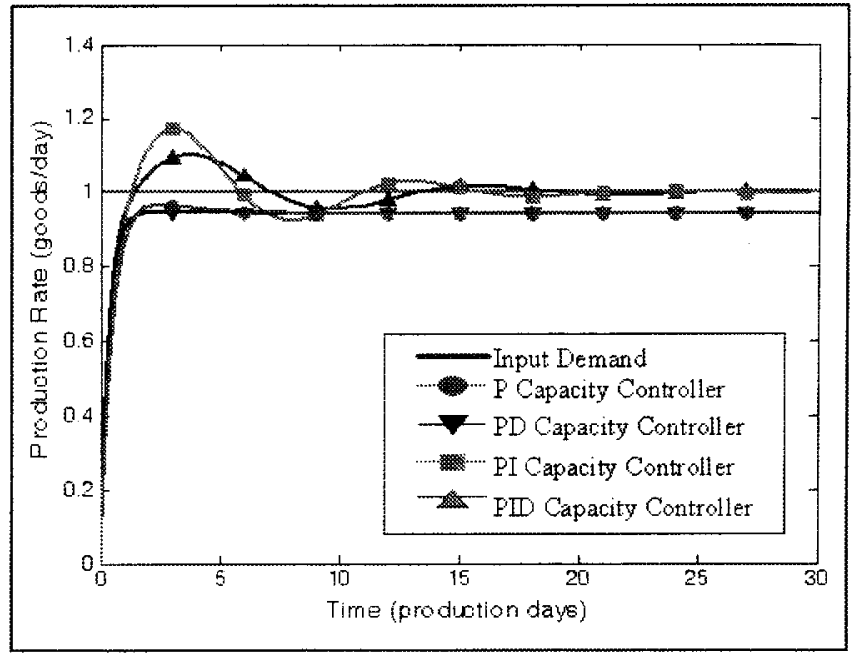

Figure 7

Comparing Different Capacity Scalability Controllers

noncontrolled capacity approach of the same line under that varying demand environment. The controller used is a proportional one with $G_{C}=4 \mathrm{~K}$ and $G_{W}=1 \mathrm{~K}$. Other types of controllers are considered in further work. The comparison is shown in Figures $8 a$ and $8 b$.

Analysis of the previous figure reveals the following points:

- The proposed capacity scalability system has a better performance under a varying demand environment, which is a typical environment of an RMS. This is shown in the required time to eliminate the backlog and also to reach the required WIP level. The uncontrolled system required 40 days to eliminate the backlog, while the controlled system required 30 days. In addition, it

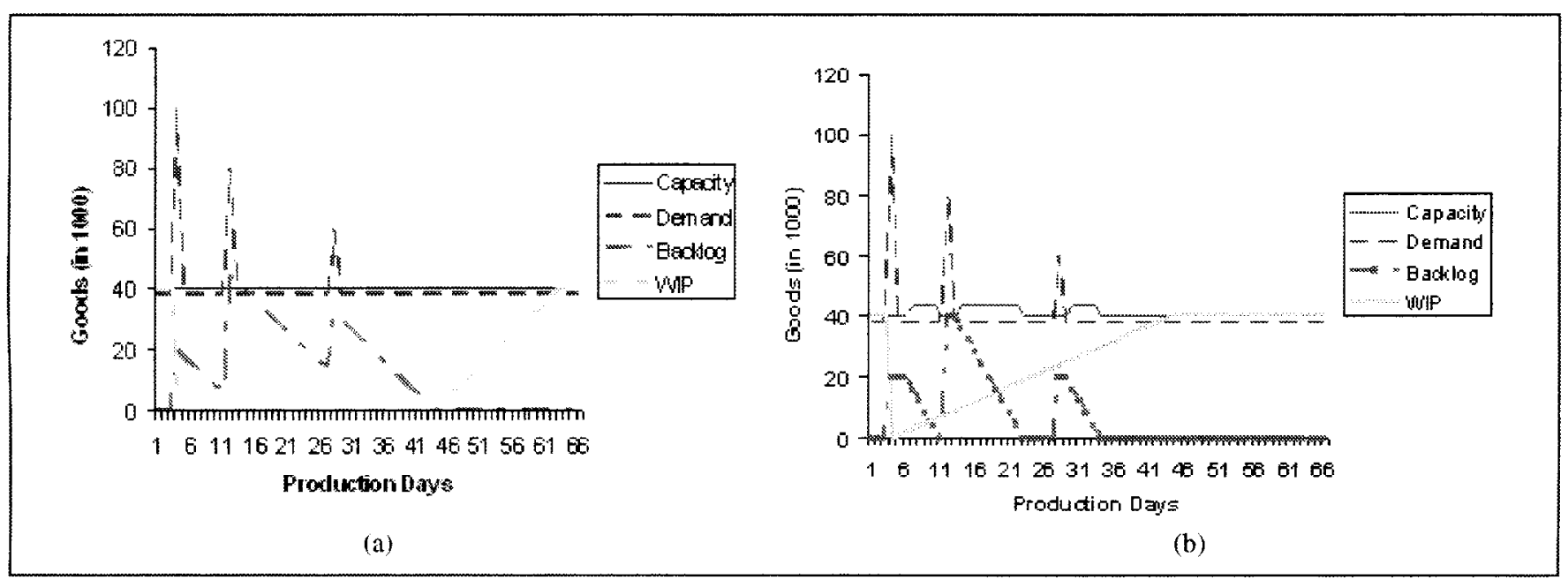

Figure 8

Capacity Scalability Under Varying Demand: (a) Uncontrolled System, (b) Controlled System 
took the uncontrolled system 60 days to maintain the target WIP level, while it took the controlled system 40 days to maintain the same level. This superior performance is due to the contribution of the engaged capacity scalability and WIP controllers in the proposed system.

- The damping effect of WIP is clear in both systems. The current WIP reduced the backlog in the first demand change in both cases. This confirms the importance of accounting for WIP to enhance the stability RMS.

\section{Conclusions and Future Work}

Reconfigurable manufacturing systems are characterized by evolution over time based on market changes. The dynamic nature of these systems dictates that they should be viewed from a dynamic standpoint. This paper presented a dynamic analysis for one of the major characteristics of these systems, which is capacity scalability. Transfer function analysis methods were used to obtain the dynamic model, which was further used to design control laws necessary to achieve the target performance of RMSs. In RMSs, it is claimed that exact capacity is supplied when needed. To examine this statement, a dynamic analysis was conducted to explore how exact capacity can be supplied and when it is needed.

To examine how capacity can be scaled when needed, the problem of capacity scalability delay was modeled by an exponential delay component in the developed RMS model. Results of the system response to market change with no delay showed that the system is more responsive. This confirms the known fact that to have successful implementation of RMS a lot of work should be done to decrease this delay and improve the ramp-up time of new configurations. An approach to achieve this goal was suggested in this paper by implementing a proportional plus a derivative, PD, controller when designing the capacity scalability controller to account for both the required capacity and the extra delay time. Results of comparing the two capacity scalability controllers (P and PD) showed a higher responsiveness to market changes when implementing the PD design in the capacity scalability controller of the dynamic RMS model. Also, the PD controller improved the overshooting of production due to this capacity scalability delay time. It was also shown that the production overshooting problem can be better managed if the capacity scalability delay time increases. This leads to the importance of having a managerial trade-off decision that balances between market responsiveness and stable production performance.

Initial investigations to examine how exact capacity scalability can be achieved showed that this is possible through eliminating production offset or drift in its dynamic response to demand changes and accounting for different delays and decisions associated with the scalability process. The paper proposed a proportional plus integral, PI, capacity scalability controller design to compensate for this production offset. To prove the preference of using this controller over the original proportional, $\mathrm{P}$, controller of the model, the dynamic response to market change was compared. Results showed that even if the proportional gains of the model controllers were raised, only the integral component in the PI capacity scalability controller can eliminate the production offset. This result is very important when speaking about maintaining a high customer service level through adopting RMSs.

The dynamic analysis of the developed model also highlighted that significant advantage is to be gained in adopting a work in process, WIP, controller in systems with capacity scalability like RMSs. WIP plays an important role in damping the production overshoot in response to market change during capacity scalability. The significant improvement in the dynamic performance of RMSs due to the augmented WIP controller in the model was shown via plotting the production response to market change with different WIP controller gain values.

It was outlined that different capacity scalability controller designs (or policies) can be adopted based on the market strategy and the specific structure of each manufacturing systems. However, a sound capacity scalability controller design that was proposed in this paper was a proportional plus integral plus derivative, PID, controller. This controller combines the merits of the two investigated controllers, PI and $\mathrm{PD}$, of eliminating the production offset and decreasing the overshooting effect of capacity scalability delay, respectively. Results showed the superiority of the PID capacity controller over other P, PI, and PD controllers in responding to market changes.

To highlight the applicability of the proposed approach, a numerical example of a machine shop under varying demand was simulated. Results showed the superiority of the proposed controlled capacity scalability system over the uncontrolled 
system in terms of time required to eliminate back$\log$ and to reach the required WIP level.

From a more practical perspective, the previous theoretical analysis needs to be properly mapped to real practice. For example, if this model represents reconfigurable parallel flow line, then $G_{C}$ would estimate the number of parallel machines to be added or removed (on a system level) and/or the number of machine modules to be added or removed (on a machine level). The integral and derivative control parameters would reflect the extra time, effort, and capacity required to reconfigure the different stages and stations of the line to meet the exact demand on time. In addition, although the model is based on continuous domain, the practical implementation would make the capacity change less frequent through slowing down the response of the capacity loop and through the delay time, $T_{D}$. However, the full practical implementation of RMSs is still a challenge due to the existing gap between research and technology development in this new field.

In conclusion, to achieve exact capacity scalability when needed in RMSs, an effort should be made to decrease the different time delays, although they cannot be totally eliminated, and to better manage the complex procedures associated with hard and soft activities of the capacity scalability process. One approach to do this is to design a proper capacity scalability controller in which each component in the design of that controller reflects the different scalability policies as discussed in this paper.

Further work is necessary to examine the effect of different design parameters of the developed dynamic model. Studying optimal settings based on performance requirements is a natural extension of this work. The assumption of having the expected production lead time equal the real production lead time should be investigated to test the effect of having a varying production lead time on capacity scalability in RMSs. Also, the behavior of the system to other kind of demand changes (not only step change) needs to be tested. Finally, more control analysis can be conducted like studying other controller design alternatives and the stability boundaries for the developed dynamic RMS model. The integration of control theoretic approaches with modern dynamic manufacturing systems analysis is indeed a potential for further research and can be used to enlighten different characteristics of these modern systems.

\section{References}

Asl, R. and Ulsoy, A. (2002a). "Capacity management in reconfigurable manufacturing systems with stochastic market demand." Proc. of ASME Int'l Mechanical Engg. Congress and Exposition.

Asl, R. and Uisoy, A. (2002b). "Capacity management via feedback control in reconfigurable manufacturing systems." Proc. of Japan-USA Symp. on Flexible Mfg. Automation, Hiroshima, Japan.

Deif, A. and ElMaraghy, W. (2006). "Effect of time-based parameters on agility of a dynamic MPC system." Annals of the CIRP (v55/1).

Deif, A. and ElMaraghy, W. (2005). "Capacity scalability in reconfigurable manufacturing systems." Proc. of CIRP 3rd Conf. for Reconfigurable Mfg. Systems, Ann Arbor, MI.

Deif, A. and ElMaraghy, W. (2004). "Reconfigurable manufacturing systems design architecture." Proc. of 14th Int'l CIRP Design Seminar, Cairo.

Disney, S. and Towill, D. (2005). "Eliminating drift in inventory and order based production control systems." Int'l Journal of Production Economics (v93-94), pp331-344.

Duffie, N. and Falu, I. (2002). "Control-theoretic analysis of a closed loop PPC system." Annals of the CIRP (v52/1), pp379-382.

Hopp, W.J. and Spearman, M.L. (1996). Factory Physics: Foundations of Manufacturing Management. Boston: Irwin/McGraw-Hill.

Hoyt, J. (1980). "Determining dynamic lead times for manufactured parts in a job shop." Computers in Manufacturing Execution and Control Systems, J.J. Pennsanken, ed. New Jersey: Auerbach Publishers, ppl-12.

John, S.; Towill, D.R.; and Naim, M. (1994). "Dynamic analysis of a WIP compensated support system." Int'l Journal of Mfg. Svstem Design (v1), pp283-297.

Kim, J-H. and Duffie. N. (2004). "Backlog control design for a closed loop PPC system." Annals of the CIRP (v53/1), pp456-459.

Leachman, R. and Carmon, T. (1992). "On capacity modeling for production planning with alternative machine types." IIE Trans. (v24, n4), pp62-72.

Liberpoulos, G. (2002), "Production capacity modeling of alternative, non-identical, flexible machines." Int'l Journal of Flexible Mfg. Systems (v14), pp345-359.

Luss, H. (1982). "Operation research \& capacity expansion problems: a survey." Operations Research (v30, n5), pp907-947.

Manne, Alan S. (1967). Investments for Capacity Expansion. Size, Location, and Time-Phasing. Cambridge. MA: MIT Press.

Mehrabi, M.G.; Ulsoy, A.G.; and Koren, Y. (2000). "Reconfigurable manufacturing systems: key to future manufacturing." Journal of Intelligent Mfg. (v11, n4), pp403-419.

Nyhuis, P. (1994). "Logistic operating curves-a comprehensive method for rating logistic potentials." EURO XIII/OR36, Univ. of Strathclyde, Glasgow.

Petermann, D. (1996). Modellbasierte Produktionsregelung (in German). Fort schritt-Berichte VDI, Dusseldorf.

Ratering, A. and Duffie. N. (2003). "Design and analysis of closedloop single-workstation PPC system." Annals of the CIRP (v52/1). pp375-379.

Roundy, R.; Zhang. F.; and Cakanyildirum, M. (2000). "Optimal capacity expansion for multi-product, multi-machine manufacturing systems with stochastic demand." Technical Report 1271. Ithaca, NY: School of Operations Research and Industrial Engg., Cornell Univ.

Simon, H. (1952). "On the application of servomechanism theory in the study of production control." Econometrica (v20. n2), pp247-268.

Son, S.Y.; Olsen. T.L.; and Yip-Hoi, D. (2001). "An approach to scalability and line balancing for reconfigurable manufacturing systems." Integrated Mfg. Systems (v12, n7), pp500-511.

Sterman, J.D. (2000). Business Dynamics: Systems Thinking and Modeling for a Complex World. New York: McGraw-Hill.

Sterman, J.D. (1989). "Modelling managerial behaviour: misperceptions of feedback in a dynamic decision-making experiment." Mgmt. Science (v35, n3), pp321-339. 
Towill, D. (1982). "Dynamic analysis of an inventory control and order-based production control system." Int'l Journal of Production Research (v20), pp671-687.

Towill, D.; Evans, G.; and Cheema, P. (1997). "Analysis of an adaptive reasonable inventory control system." Journal of Production Planning \& Control (v8, n6), pp545-557.

Urbani, A.; Molinari-Tosatti, L.; Pedrazzoli, P.; Fassi, 1.; and Boer, C.R. (2001). "Flexibility and reconfigurability: an analytical approach and some examples." CIRP 1st Conf. for Reconfigurable Manufacturing Systems, Ann Arbor, MI.

Wiendahl, H-P. (1995). Load-Oriented Manufacturing Control. Berlin: Springer-Verlag.

Wiendahl, H-P. and Breithaupt, J-W. (1999). "Modelling and controlling the dynamics of production systems." Journal of Production Planning \& Control (v10, n4), pp389-401.

Wiendahl, H-P. and Breithaupt, J-W. (2000). "Automatic production control applying control theory." Int'l Journal of Production Economics (v63, n1), pp33-46.

Wikner, J. (1994). "Dynamic modeling and analysis of information flows in production-inventory and supply chain systems." Pro. 1 Series No. 12. Linkoping Institute of Technology, Sweden.

Wikner, J. (2003). "Continuous-time dynamic modeling of variable lead time." Int'l Journal of Production Research (v41, n12), pp2787-2798.

\section{Authors' Biographies}

Dr. Ahmed Deif successfully defended his PhD dissertation in the Dept. of Industrial and Manufacturing Systems Engineering (IMSE) at the University of Windsor in December 2006. He received his MS from the University of Windsor (2003) and his BSc from The American University in Cairo (1999). His research interests are in manufacturing capacity management and dynamic analysis of manufacturing systems.

Dr. Waguih ElMaraghy is professor of engineering and Director of the Intelligent Manufacturing Systems (IMS) Centre, Dept. of Industrial and Manufacturing Systems Engineering, University of Windsor. $\mathrm{He}$ is also a senior member of the Society of Manufacturing Engineers (SME), the Society of Automotive Engineers (SAE), a Fellow of the Canadian Society for Mechanical Engineering (CSME), a Fellow of the American Society of Mechanical Engineers (ASME), and a member of the CIRP Academy (International Institute for Production Engineering Research). Dr. ElMaraghy is an active researcher and author with expertise in engineering design. manufacturing automation, and industrial productivity.

\title{
Appendix
}

\author{
Dynamic RMS Model Notations: \\ WIP* Desired WIP level (parts) \\ WIP Actual WIP level (parts) \\ DPR Desired production rate (parts/days) \\ PR Actual production rate (parts/days) \\ $T_{L T}^{*} \quad$ Expected lead time (days) \\ TLT Lead time (days) \\ GW WIP control gain (1/day) \\ Cap* Desired capacity rate (parts/days) \\ $G C \quad$ Capacity scalability control gain (parts/days) \\ TD Capacity installation delay time (days) \\ OR Expected order rate (parts/days)
}

